\title{
Connections between Retinex, neural models and variational methods
}

\author{
Marcelo Bertalmío, Universitat Pompeu Fabra, Spain
}

\begin{abstract}
This paper explores the interrelations between Retinex, neural models and variational methods by making an overview of relevant related works in the past few years. Taking all the essential elements of the Retinex theory as postulated by Land and McCann (channel independence, the ratio reset mechanism, local averages, non-linear correction), it has been shown that we can obtain a Retinex algorithm implementation that is intrinsically $2 D$, whose results comply with all the expected properties of the original, one-dimensional path-based Retinex algorithm (such as approximating color constancy while being unable to deal with overexposed images) but don't suffer from common shortcomings such as sensitivity to noise, appearance of halos, etc. An iterative application of this $2 D$ Retinex algorithm takes the form of a partial differential equation (PDE) that it's proven not to minimize any energy functional, and this fact is linked to its limitations regarding over-exposed pictures. It was proposed to modify in this regard the iterative method in a certain way so that it is able to handle both under and over-exposed images, the resulting $P D E$ now has a number of very relevant properties which allow to connect Retinex with variational models, histogram equalization and efficient coding, perceptual color correction algorithms, and computational neuroscience models of cortical activity and retinal models.
\end{abstract}

\section{The Retinex theory and the Retinex algorithm}

Land makes in [21] a very clear and detailed explanation of his Retinex theory and the experiments that led him to its postulation. An experiment carried out by Land and his team consisted of arranging colored sheets of paper into two identical panels (socalled "Mondrians"); quoting from [21]:

"Each 'Mondrian' is illuminated with its own set of three projector illuminators equipped with band-pass filters and independent brightness controls so that the long-wave ('red'), middle-wave ('green') and short-wave ('blue') illumination can be mixed in any desired ratio. A telescopic photometer can be pointed at any area to measure the flux, one wave-band at a time, coming to the eye from that area. [...] In a typical experiment the illuminators can be adjusted so that the white area in the Mondrian at the left and the green area (or some other area) in the Mondrian at the right are both sending the same triplet of radiant energies to the eye. Under actual viewing conditions white area continues to look white and green area continues to look green even though the eye is receiving the same flux triplet from both areas."

A variant of this experiment was a color-matching test, where the observer was presented simultaneously with the colored-sheet panel under some experimental illuminant (this was seen through the left eye), and a standard chart of color patches, the Munsell Book of Color, under a "white" iluminant (seen through the right eye). In each run of the test the observer was asked to look at a given colored sheet on the left and select the Munsell patch of the right that best matched its color. The experimental illuminant was adjusted in each run so that the reference color sheet always sent the same radiance to the eye, regardless of its reflectance: gray, red, yellow, blue, and green sheets of paper were sending the same radiant energy and hence would be deemed identical by a photometer. But in each and every case, the observer was able to match the colored sheet to the closest Munsell patch, i.e. red sheet to red patch, green sheet to green patch, etc. In each matching pair, sheet and patch had different radiances but the same (scaled) integrated reflectance, which is defined, for each waveband, as a ratio: the integral of the radiance of the object (sheet or patch) over the waveband, divided by the integral over the same waveband of the radiance of a white object under the same illuminant. The scaling is a non-linear function that relates reflectance to lightness sensation.

Land's conclusion was that color perception had a physical correlate in these scaled integrated reflectances, which implies that in order to perceive the color of an object somehow our visual system is comparing the light coming from the object with the light coming from a reference white, since both magnitudes are needed to compute the ratio of the integrated reflectances. He wondered how we are able to find this reference white "in the unevenly light world without reference sheets of white paper" [21]. The sensation of white will be generated by the area of maximum radiance in all three bands (this is the von Kries' model or "white-patch" assumption); this area could be used as reference, but Land didn't know how our visual system could "ascertain the reflectance of an area without in effect placing a comparison standard next to the area" [21]. The solution he proposed consisted of comparing far-away points through paths: the ratio of the values at the two end-points of the path can be computed as the sequential product of the ratios of each point of the path with the following point. This sequential product is reset to 1 whenever a point is reached where the value is larger than the previous maximum along the path. In this way, the sequential product for a path provides the ratio between the value at an end-point and the maximum value of the path, which is the estimate for "reference white" that can be obtained with the given path. This is the "ratio-reset" mechanism. The Retinex algorithm consists of assigning, for each point and each waveband (long, middle, short), an estimate reflectance obtained as the average of the sequential products obtained on many paths, all ending in the given point. Land thought that this was a plausible explanation of how our visual system estimates reflectances but he didn't want to venture where exactly this type of operations were being carried out, in the retina or at the cortex; therefore he chose the name "Retinex" for his approach. 
The Retinex algorithm can be directly applied to digital images in a straightforward way, where the pixels will be the points and the three color channels R, G and B will be the wavebands. Over the years a great number of works have been published following and/or adapting the original formulation; see Bertalmío et al. [3] for an overview of the most relevant ones and Provenzi et al. [31] for a detailed mathematical description of the Retinex algorithm. As proved in [31], Retinex always increases brightness so it can't be directly applied to overexposed pictures; also, if the algorithm is iterated the results may improve but the convergence image is flat white, so there is some "sweet spot" of the number of iterations yielding the best output [3]. Another major source of problems is Retinex's reliance on paths: their length, shape and number condition the results and many works have been proposed trying to optimize the selection of these variables.

\section{ACE}

The Automatic Color Enhancement (ACE) algorithm of Rizzi et al. [32] is also based on perception, and its relationship with Retinex will become clear shortly.

$\mathrm{ACE}$ is designed to mimic some basic characteristics of the human visual system, like the white patch and the grey world mechanisms, lateral inhibition, the independence of chromatic channels, or the influence of spatial relationships in the scene. It consists of two stages, which are applied to each channel independently:

- In the first stage, given an input one-channel image $I$, an intermediate output image $R$ is computed in this way:

$$
R(p)=\frac{1}{M} \sum_{j} \frac{r(I(p)-I(j))}{d(p, j)},
$$

where $p, j$ are pixels, $r(x)$ is a non-linear, sigmoid function going from -1 when $x \rightarrow-\infty$ to +1 when $x \rightarrow+\infty$ and taking the value 0 at 0 , and $d()$ is a distance function whose value decreases as the distance between $p$ and $j$ increases. The value $M$ is a normalization constant ensuring that the maximum of $R$ is 1 , therefore $R$ is always in the range $[-1,1]$. This stage considers the basic principles mentioned above of chromatic independence, lateral inhibition, and spatial influence.

- In the second stage, the final output image $O$ is computed as

$$
O(p)=255 \times\left(\frac{1}{2}+\frac{R(p)}{2}\right) .
$$

This operation performs a simultaneous white patch and grey world scaling: white patch because a pixel $p$ with maximum value $R(p)=1$ will have an output $O(p)=255$, and gray world because an average gray pixel $p$ should have a value $R(p)=0$ and therefore an output $O(p)=127.5$.

The authors perform experiments that show how ACE has several excellent properties: it allows to obtain very good color constancy, it increases the dynamic range of the input and, unlike Retinex, it can deal both with under- and over-exposed pictures, it can perform de-quantization (eliminating quantization artifacts produced by encoding an image with an unsufficient number of bits per channel), and it can reproduce some visual perception ilusions. Its main limitation is its computational complexity, $O\left(N^{2}\right)$ where $N$ is the number of pixels.
It also raises several interesting questions: what, if any, is its relationship with Retinex? If iterated, does it produce different results? And if this is the case, do they converge? We shall answer these questions in the following sections.

\section{Image processing for contrast enhancement: histogram equalization}

Histogram equalization is a classical, very basic image processing technique dating at least to the early 1970s (see [30] and references therein), aiming at enhancing the contrast and improving the appearance of images by way of re-distributing their levels uniformly accross the available range. In this sense, an image would be optimal if its histogram were flat or "equalized", meaning that all the range is used and all levels are represented by the same amount of pixels. Therefore, when an image has a flat histogram its cumulative histogram is simply a ramp, and this allows for a very straightforward computation for the histogram equalization procedure: assuming we are working on a graylevel image in the range [0,1], we have to substitute each level $g$ in the original image by the value of its normalized cumulative histogram, $H(g)$. The solution is computed very fast using a look-up table (LUT).

While histogram equalization may improve the visual appearance of an image, in many situations the end result may look worse than the original, often when the image is overexposed [30]. This is aggravated by the fact that the equalization procedure is a one-shot technique, that only produces a final result, without any "in-between", so if the resulting image shows any type of unpleasant artifact there is nothing to do about it. This issue was addressed by Sapiro and Caselles [34], who proved that the minimization of the energy functional

$$
E(I)=2 \sum_{x}\left(I(x)-\frac{1}{2}\right)^{2}-\frac{1}{A B} \sum_{x} \sum_{y}|I(x)-I(y)|
$$

produces an image $I$ with a flat histogram. The range of $I$ is $[0,1]$, $x, y$ are pixels and $A, B$ are the image dimensions. While the result of histogram equalization is very often unsatisfactory and can't be altered, [34] proposes to start with an input image $I_{0}$ and apply to it step after step of the minimization of Equation 3, letting the user decide when to stop. If the user lets the minimization run to convergence, she'll get the same result as with a LUT, but otherwise a better result can be obtained if the iterative procedure stops before the appearance of severe artifacts. The squared differences in the first term of Equation 3 and the absolute differences in the second one are required to ensure that the minimization yields an image with equalized histogram, see [34] for details. The energy in Equation 3 can be interpreted as the difference between two positive and competing terms,

$$
E(I)=D(I)-C(I)
$$

The first term measures the dispersion around the average value of $\frac{1}{2}$, as in the gray world hypothesis for color constancy, stating that our visual system estimates the illuminant as one half the average of the colors of the scene, an observation made by $[12,13]$ and formalized by [9]. The second term measures the contrast as the sum of the absolute value of the pixel differences.

\section{Perceptually-based contrast enhancement}

The abovementioned measure of contrast is global, not local, i.e. the differences are computed regardless of the spatial 
locations of the pixels. This is not consistent with how we perceive contrast, which is in a localized manner, at each point having neighbors exert a higher influence than far-away points. Using the concepts introduced by the popular perceptually-based color correction method ACE of [32], the authors of [4] propose an adapted version of the functional of Equation 3 that complies with some very basic visual perception principles, namely those of locality, color constancy and white patch (the latter stating that the brightest spot in the image is perceived as white, an observation which has a long history that dates back at least to the works of Helmholtz, as explained by $[14,15])$ :

$$
\begin{array}{r}
E(I)=\frac{\alpha}{2} \sum_{x}\left(I(x)-\frac{1}{2}\right)^{2}-\gamma \sum_{x} \sum_{y} w(x, y)|I(x)-I(y)| \\
+\frac{\beta}{2} \sum_{x}\left(I(x)-I_{0}(x)\right)^{2},
\end{array}
$$

where $w$ is a distance function such that its value decreases as the distance between $x$ and $y$ increases, $I_{0}$ is the original image and $\alpha, \beta$ and $\gamma$ are positive weights (which can be chosen so as to guarantee the white patch property, see [4] for details). The gradient descent equation for the functional in Equation 5 is the following:

$$
\begin{array}{r}
I_{t}(x)=-\alpha\left(I(x)-\frac{1}{2}\right)+\gamma \sum_{y} w(x, y) \\
\operatorname{sgn}(I(x)-I(y)) \\
-\beta\left(I(x)-I_{0}(x)\right) .
\end{array}
$$

Starting from $I=I_{0}$, we iterate Equation 6 until we reach a steady state, which will be the result of this algorithm.

By minimizing the energy in Equation 5 we are locally enhancing contrast (second term) and promoting color constancy by discounting the illuminant (first term), while preventing the image from departing too much from its original values (third term). We could also say that the minimization of Equation 5 approximates local histogram equalization.

In [4] it is shown that Eq. 5 has a single minumum and that the image $I$ minimizing Eq. 5 is a fixed point of ACE. In other words, we can say that ACE is a numerical implementation of the gradient descent of Eq. 5, and this answers one of the questions raised in the previous section, namely, that iterating ACE we do obtain different results. Also, this iterative procedure converges (because there is a unique minimum), but for this we need $\beta>0$.

The minimization of Eq. 5 yields very good color constancy results and this method shares all the good properties and possible applications of ACE, plus the numerical implementation in [4] has a reduced complexity of $O(N \log N)$, where $N$ is the number of pixels.

Apart from the color constancy application, this method can be used for contrast enhancement as well, since it produces good results without halos, spurious colors or any other kind of visual artifact.

There is a very close connection between this formulation and Retinex, which we will present in the following section.

\section{Connections between Retinex, ACE and vari- ational models}

In their kernel-based Retinex (KBR) formulation, Bertalmío et al. [3] take all the essential elements of the Retinex theory as presented in [21] (channel independence, the ratio reset mechanism, local averages, non-linear correction) and propose an implementation that is intrinsically $2 \mathrm{D}$, and therefore free of the issues associated with the 1D paths used in the original Retinex algorithm. The results obtained with this algorithm comply with all the expected properties of Retinex (such as performing color constancy while being unable to deal with overexposed images) but don't suffer from the usual shortcomings such as sensitivity to noise, appearance of halos, etc. In [3] it is proven that there isn't any energy that is minimized by the iterative application of the KBR algorithm, and this fact is linked to its limitations regarding overxposed pictures. Using the analysis of contrast performed by Palma-Amestoy et al. [28], in [3] it is determined how to modify the basic KBR equation so that it can also handle overexposed images, and the resulting, modified KBR equation turns out to be essentially the gradient descent of the energy given by Equation 5. In other words, the method of [4] can be seen as an iterative application of Retinex, although in a modified version that allows to produce good results also in the case of overexposed images.

In this way, the connection between Retinex, ACE, and the variational method of [4] becomes explicit.

\section{Connections with neuroscience Efficient coding}

As pointed out in [6], the human visual system works in many ways in order to efficiently encode the visual information coming from natural environments, reducing its inherent redundancy, as proposed in the seminal work of [2] (see [27] for a review). For instance, while natural scenes have luminance distributions which are very lopsided, with a high peak and a very rapid fall-off, photoreceptors encode this information with signals that have a much more even distribution: indeed, photoreceptors perform histogram equalization, as demonstrated by [22]. And the receptive fields of visual neurons, both retinal and post-retinal, compensate the $1 / f^{2}$ decay of the power spectrum of natural images, whitening the spectrum of the resulting signal and thus minimizing interpixel redundancies and increasing coding efficiency (see [1], and [10] where the existence of whitening at the local geniculate nucleus is demonstrated for natural images).

Apart from efficiency in coding, another very important aspect is that of biological efficiency in terms of wiring. The resolution of retinal mosaics is limited by the number of axons that can pass through the optic nerve, which acts as a bottleneck ([26]). But the visual system is able to achieve a visual acuity beyond the limit imposed by the number of photoreceptors at the retina: in their classical paper on contrast constancy, [11] suggest that there are cortical mechanisms for contrast enhancement that compensate for the blurring produced in early stages of the visual process. Very recently [25] it has been confirmed that contrast enhancement takes place at the lateral geniculate nucleus (LGN) and, remarkably, the authors point out that this contrast enhancement procedure is very much alike the common techniques used in image processing.

The formulations stated above on energy minimization, histogram equalization and efficient coding let us make an important connection between the variational model of [4] and neuroscience. The activity of a population of neurons in the region $V 1$ of the visual cortex evolves in time according to the WilsonCowan equations $[8,38,39]$. Treating $V 1$ as a planar sheet of ner- 
vous tissue, the state $a(r, \phi, t)$ of a population of cells with cortical space coordenates $r \in R^{2}$ and orientation preference $\phi \in[0, \pi)$ can be modeled with the following PDE [8]:

$$
\begin{aligned}
& \frac{\partial a(r, \phi, t)}{\partial t}=-\alpha a(r, \phi, t)+ \\
& +\mu \int_{0}^{\pi} \int_{R^{2}} \omega\left(r, \phi \| r^{\prime}, \phi^{\prime}\right) \sigma\left(a\left(r^{\prime}, \phi^{\prime}, t\right)\right) d r^{\prime} d \phi^{\prime}+h(r, \phi, t)
\end{aligned}
$$

where $\alpha, \mu$ are coupling coefficients, $h(r, \phi, t)$ is the external input (visual stimuli), $\omega\left(r, \phi \| r^{\prime}, \phi^{\prime}\right)$ is a kernel that decays with the differences $\left|r-r^{\prime}\right|,\left|\phi-\phi^{\prime}\right|$ and $\sigma$ is a sigmoid function. If we ignore the orientation $\phi$ and assume that the input $h$ is constant in time, it can be shown that Equation 7 is closely related to the gradient descent of Eq. 5, where neural activity $a$ plays the role of image value $I$, sigmoid function $\sigma$ behaves as the derivative of the absolute value function, and the visual input $h$ is the initial image $I_{0}$. Therefore the variational model of [4], based on the efficient coding principle of (local) histogram equalization, shows close similarities with the classical neural model of Wilson and Cowan. As Bertalmío and Cowan [5] point out, this suggests that the Wilson-Cowan equations are the gradient descent of a certain energy, and also that there would appear to be a physical substrate at the cortex for the Retinex model.

\section{Lightness induction}

Alongside mechanisms for coding and wiring efficiency, there is neural activity in region $V 1$ of the human visual cortex that correlates with the perceptual phenomenon of lightness induction, as proven by [29]. The term lightness induction or achromatic induction designates the visual phenomenon by which the perceived reflectance of an object depends on its surround. It can take the form of lightness contrast, when the object's lightness shifts away from that of its surroundings: a dark object on a light background appears even darker, or a light object in a dark surround becomes even lighter. The reverse is called lightness assimilation, in which case the appearance of the object shifts in the direction of the lightness of its surround. Shevell [35] mentions that lightness assimilation occurs in situations of high spatial frequency while lightness contrast is associated with relatively lower spatial frequencies. Looking closely at Equation 6, we can see that the spatial arrangement of the image data plays no role in it. Therefore, we can expect that the local contrast enhancement procedure of [4] will always produce lightness contrast, not assimilation.

Rudd [33] studies lightness induction using a display with two disk-and-ring (DAR) stimuli over a uniform background for matching experiments. The intensity of the background $B$, of the left ring $R_{M}$, and of the disk on the right $D_{T}$ is kept constant; the intensity of the right ring $R_{T}$ is modified, and the observer has to adjust the intensity of the left disk $D_{M}$ so as to match the appearance of the right disk $D_{T}$. While the data from the perceptual experiments in [33] shows a cuadratic relationship (inverted parabolas) between $R_{T}$ and $D_{M}$, the model of [4] predicts a linear relationship instead (straight lines with negative slope).

In order to overcome these intrinsic limitations of the method in [4] with respect to lightness induction, apparently some notion of spatial frequency should appear in the energy functional. Bertalmío [6] proposes a new model consisting in the following
PDE, a modification of the gradient descent equation (6):

$$
\begin{aligned}
& I_{t}(x)=-\alpha(I(x)-\mu(x))+ \\
& +\gamma\left(1+(\sigma(x))^{c}\right) \sum_{y} w(x, y) \operatorname{sgn}(I(x)-I(y)) \\
& \quad-\beta\left(I(x)-I_{0}(x)\right),
\end{aligned}
$$

where $\mu(x)$ is the mean average of the original image data computed over a neighborhood of $x, \sigma(x)$ is the standard deviation of the image data computed over a small neighborhood of $x$, and the exponent $c$ is a positive constant. The differences with respect to Equation 6 are that now the average in the first term is no longer global (the 1/2 value of Equation 6) but local, and that the weight for the second term is no longer a constant, but it changes both spatially and with each iteration, according to the local standard deviation $\sigma$ : if the neighborhood over which it is computed is sufficiently small, standard deviation can provide a simple estimate of spatial frequency. Also, the standard deviation is commonly used in the vision literature as an estimate of local contrast. This contrast $\sigma(x)$ is raised to a power $c$, which is also the case with other neural models such as [24] and [16] where a power law is applied to the contrast. This new model is able to predict perceptual phenomena like the lightness induction results of Rudd [33]: the predicted match luminance plots are no longer linear but quadratic, with an initial lightness assimilation regime for low values of $R_{T}$ followed by a lightness contrast part, and the curvature of these parabolas decreases with increasing ring width.

But while the results of [6] now show both assimilation and contrast, the modification in the model may appear as ad-hoc changes, not easy to reconcile with the basic postulates of Wilson and Cowan's theory. In a later work [7], a Wilson-Cowan type of equation is proposed that slightly modifies the model in [4] and is capable to predict visual induction results:

$$
\begin{aligned}
I_{t}(x) & =-\alpha(I(x)-\mu(x))+ \\
& +\gamma \sum_{y} w(x, y) \operatorname{sgn}(I(x)-I(y))-\beta\left(I(x)-I_{0}(x)\right),
\end{aligned}
$$

where the target global mean average $1 / 2$ that appeared in [4] is replaced by $\mu(x)$, which is a local average of the image value $I$ computed by convolution with a kernel obtained as a weighted sum of two Gaussians of different standard deviation.

\section{Retinal models}

Regarding induction, Kim and Bertalmío note in $[19,20]$ that while contrast induction is attributed to a local-range lateral inhibition in the retina, there is little consensus on a neural structure underlying assimilation. Some studies postulated that assimilation occurs by an unknown post-retinal mechanism that performs long-range surface interaction. However, in [19] it is proposed that such long-range mechanism exists in the retina based on recent neurophysiological evidence that the main retinal inhibitory feedback interneurons manifest wide receptive-fields (RFs). The work cross- examined the effect of these wide RFs in two different biophysical retinal model platforms $[36,37,40]$ and confirmed that the cell responses in both of the models match to behavioral data of the brightness contrast and assimilation as a function of surrounding surface size, if and only if the wide RFs are considered. This would appear to be the first evidence in the literature 
that the wide RFs of inhibitory interneurons serve for long-range surface interaction, that local contrast and long-range surface interaction share the same neural locus, and that brightness assimilation is inaugurated at the retinal level.

The properties of human contrast perception show a close correlation to the responses of retinal ganglion cells [17, 23], whose spatial processing properties (the isotropic center-surround processing) are shaped by the local feedback from interneurons (horizontal / amacrine cells) to the feed-forward cells (photoreceptor, and bipolar / ganglion cells). In [18] the authors study the computational structure of this retinal feedback system. They start identifying a simple form of a system of differential equations that realizes the retinal feedback architecture and analyzed its steady-state behaviour to a static stimulus input (i.e. model cell voltage responses equilibrated to the input). Three main conclusions may be derived from the results of the analysis. Firstly, the system of equations preserves the ability to predict some human contrast perception properties such as spatial-frequency dependent contrast sensitivity and brightness induction, and provides further support to the possibility that brightness assimilation starts at the retina. Secondly, the steady-state response of the system can be obtained in a single pass by convolving the original input with a single kernel (a combined product of different extents of receptive-fields of the retinal cells) and therefore [18] proposes a computationally efficient way of modeling retinal cell responses and the resulting human contrast perception. Finally, finding the steady state solution is mathematically equivalent to solving an optimization problem of maximizing the spatial contrast in the encoded signals while being faithful to the local light intensity of the input stimulus, which is remarkably similar to the minimization of the energy functional of Eq. 5 proposed in [4]. This provides interesting connections with efficient coding theories and computational neuroscience models like the ones discussed above, as well as suggesting a biologically plausible realization, at retinal level, of the "ratio-reset" mechanism of the Retinex algorithm.

\section{Conclusion}

In this work we have made an overview of recent works from the image processing and visual sciences literature that allow to connect Retinex with variational models, histogram equalization and efficient coding, perceptual color correction algorithms, and computational neuroscience models of cortical and retinal activity. Many key questions remain unanswered, including the exact nature of a possible biological correlate for the Retinex mechanism, but the author believes that the existence of these similarities and points in common on this subject among very different disciplines can only be beneficial for gaining understanding in all of them.

\section{Acknowledgements}

This work was supported by the European Research Council, Starting Grant ref. 306337, by the Spanish government, grant ref. TIN2012-38112, and by the Icrea Academia Award.

\section{References}

[1] J.J. Atick. Could information theory provide an ecological theory of sensory processing? Network: Computation in neural systems, 3(2):213-251, 1992.

[2] Horace B Barlow. Possible principles underlying the trans- formation of sensory messages. Sensory communication, pages 217-234, 1961.

[3] M. Bertalmío, V. Caselles, and E. Provenzi. Issues about retinex theory and contrast enhancement. International Journal of Computer Vision, 83(1):101-119, 2009.

[4] M. Bertalmío, V. Caselles, E. Provenzi, and A. Rizzi. Perceptual color correction through variational techniques. IEEE Transactions on Image Processing, 16:1058-1072, 2007.

[5] M. Bertalmío and J. Cowan. Implementing the Retinex algorithm with Wilson-Cowan equations. Journal of Physiology, Paris, 2009.

[6] Marcelo Bertalmío. From image processing to computational neuroscience: a neural model based on histogram equalization. Frontiers in computational neuroscience, 8, 2014.

[7] Marcelo Bertalmío. Modeling lightness induction with wilson-cowan equations. Journal of Vision, 14(15):56-56, 2014.

[8] P.C. Bressloff, J.D. Cowan, M. Golubitsky, P.J. Thomas, and M.C. Wiener. What Geometric Visual Hallucinations Tell Us about the Visual Cortex. Neural Computation, 14(3):473-491, 2002.

[9] G. Buchsbaum. A spatial processor model for object colour perception. Journal of the Franklin Institute, 310:337-350, 1980.

[10] Yang Dan, Joseph J Atick, and R Clay Reid. Efficient coding of natural scenes in the lateral geniculate nucleus: experimental test of a computational theory. The Journal of Neuroscience, 16(10):3351-3362, 1996.

[11] M.A. Georgeson and G.D. Sullivan. Contrast constancy: deblurring in human vision by spatial frequency channels. The Journal of Physiology, 252(3):627-656, 1975.

[12] D.B. Judd. Hue, saturation, and lightness of surface colors with chromatic illumination. Journal of the Optical Society of America, 30(1):2-32, 1940.

[13] D.B. Judd. Contributions to color science, volume 545, chapter The unsolved problem of color perception, pages 516-522. National Bureau of Standards (USA), 1979.

[14] D.B. Judd. Contributions to color science, volume 545, chapter Appraisal of Land's work on two-primary color projections, pages 471-486. National Bureau of Standards (USA), 1979.

[15] D.B. Judd. Contributions to color science, volume 545, chapter Color appearance, pages 539-564. National Bureau of Standards (USA), 1979.

[16] K.N. Kay, J. Winawer, A. Mezer, and B.A. Wandell. Compressive spatial summation in human visual cortex. Journal of neurophysiology, 110(2):481-494, 2013.

[17] DH Kelly. Visual contrast sensitivity. Journal of modern optics, 24(2):107-129, 1977.

[18] Jihyun Kim, Thomas Batard, and Marcelo Bertalmío. Retinal processing optimizes contrast coding. Journal of vision (submitted), 2016.

[19] Jihyun Kim and Marcelo Bertalmío. Investigating the effect of lateral inhibition in the retinal circuitry on lightness contrast and assimilation: A model study. Journal of vision, 15(12):633-633, 2015.

[20] Jihyun Kim and Marcelo Bertalmío. Surrounding size effect 
on brightness contrast-to-assimilation is predicted in retina. Journal of vision (submitted), 2016.

[21] E.H. Land. The Retinex theory of color vision. Scientific American, 237:108-128, 1977.

[22] S.B. Laughlin. A simple coding procedure enhances a neurons information capacity. Z. Naturforsch, 36(910-912):51, 1981.

[23] Barry B Lee, Joel Pokorny, Paul R Martin, Arne Valbergt, and Vivianne $\mathrm{C}$ Smith. Luminance and chromatic modulation sensitivity of macaque ganglion cells and human observers. JOSA A, 7(12):2223-2236, 1990.

[24] V. Mante, V. Bonin, and M. Carandini. Functional mechanisms shaping lateral geniculate responses to artificial and natural stimuli. Neuron, 58(4):625-638, 2008.

[25] L.M. Martinez, M. Molano-Mazón, X. Wang, F.T. Sommer, and J.A. Hirsch. Statistical wiring of thalamic receptive fields optimizes spatial sampling of the retinal image. Neuron, 81(4):943-956, 2014.

[26] B.A. Olshausen. Principles of image representation in visual cortex. The visual neurosciences, pages 1603-1615, 2003.

[27] B.A. Olshausen and D.J. Field. Vision and the coding of natural images. American Scientist, 88(3):238-245, 2000.

[28] R. Palma-Amestoy, E. Provenzi, M. Bertalmío, and V. Caselles. A perceptually inspired variational framework for color enhancement. IEEE Transactions on Pattern Analysis and Machine Intelligence, 31(3):458-474, 2009.

[29] M. Pereverzeva and S.O. Murray. Neural activity in human v1 correlates with dynamic lightness induction. Journal of Vision, 8(15):8, 2008.

[30] W.K. Pratt. Digital Image Processing, 4th edition. John Wiley \& Sons, 2007.

[31] E. Provenzi, L. De Carli, A. Rizzi, and D. Marini. Mathematical definition and analysis of the Retinex algorithm. Journal of the Optical Society of America A, 22(12):26132621, December 2005.

[32] A. Rizzi, C. Gatta, and D. Marini. A new algorithm for unsupervised global and local color correction. Pattern Recognition Letters, 24:1663-1677, 2003.

[33] M.E. Rudd. How attention and contrast gain control interact to regulate lightness contrast and assimilation: A computational neural model. Journal of vision, 10(14), 2010.

[34] G. Sapiro and V. Caselles. Histogram modification via differential equations. Journal of Differential Equations, 135:238-266, 1997.

[35] S.K. Shevell. The Science of Color, chapter Color appearance, pages 149-190. Elsevier Science Ltd, 2003.

[36] Hans van Hateren. A cellular and molecular model of response kinetics and adaptation in primate cones and horizontal cells. Journal of vision, 5(4):5, 2005.

[37] JH Van Hateren. A model of spatiotemporal signal processing by primate cones and horizontal cells. Journal of Vision, 7(3):3, 2007.

[38] H.R. Wilson and J.D. Cowan. Excitatory and inhibitory interactions in localized populations of model neurons. Biophysical journal, 12:1-24, 1972.

[39] H.R. Wilson and J.D. Cowan. A mathematical theory of the functional dynamics of cortical and thalamic nervous tissue. Biological Cybernetics, 13(2):55-80, 1973.

[40] Hugh R Wilson. A neural model of foveal light adaptation and afterimage formation. Visual neuroscience, 14(03):403423, 1997.

\section{Author Biography}

Marcelo Bertalmio received the Ph.D. degree in electrical and computer engineering from the University of Minnesota in 2001. He is an Associate Professor at University Pompeu Fabra in Barcelona, Spain. His interests are Image Processing and Computer Vision for digital cinema applications, although he prefers the (analog) films of Luis Buñuel and Luis García Berlanga. 